\title{
Consumo de tabaco y relaciones familiares y amicales en estudiantes de sexo femenino de la carrera de farmacia
}

\author{
Danjoy León Delia ${ }^{1, a, b}$, Cerna Silva Carolina ${ }^{1, a, c ; 2, d, ~ M u s a y o n ~ O b l i t a s ~ Y e s e n i a ~ 1, a, e ~}$
}

\section{RESUMEN}

Objetivos: determinar el consumo de tabaco, las relaciones familiares y amicales en mujeres estudiantes de farmacia. Material y métodos: estudio cuantitativo, analítico-correlacional, de corte transversal. La muestra fue extraída de la base de datos de una investigación titulada: "Conocimientos y prácticas de consumo de tabaco y otras drogas en estudiantes mujeres de farmacia", después de aplicar los criterios de selección quedo una muestra de 176 estudiantes, el instrumento fue una versión adaptada del cuestionario del Global Youth Tobacco Survey (GYTS), elaborado por OMS-CDC-CEDRO el año 2000, se usaron 42 reactivos. Las variables fueron tratadas en términos de frecuencia y porcentaje, se evaluó la asociación entre las variables relaciones familiares y amicales con el consumo de tabaco mediante Chi-cuadrado. Se usó el coeficiente de Spearman para calcular la significancia de correlación entre las puntuaciones asignadas a las relaciones familiares y amicales con la variable consumo de tabaco. Resultados: no existe una correlación significativa entre el tipo de relaciones familiares y el consumo de tabaco en estudiantes de farmacia. Las estudiantes cuyas amistades no fuman, solo un $48 \%$ fumaba, mientras que las estudiantes con algunas amistades que fumaban y aquellas que la mayoría de sus amistades fumaba, esta cifra de consumo ascendió a 70,4\% y 85,7\% respectivamente. Conclusiones: no existe una correlación significativa entre el tipo de relaciones familiares y consumo de tabaco en estudiantes de farmacia. Si existe una relación significativa entre el consumo de tabaco en estudiantes de farmacia y que algunas o la mayoría de sus amistades fumen.

PALABRAS CLAVE: consumo asociado, tabaco, estudiantes, mujeres, farmacia.

\section{Consumption of snuff and family and friendly relations in female students of pharmacy career}

\section{SUMMARY}

Objectives: to determine the consumption of snuff, family and women friendly relations pharmacy students. $\mathbf{M a}$ terial and Methods: quantitative, analytical and correlational, cross-sectional study. The sample was drawn from the database of research entitled "Knowledge and practices of consumption of snuff and other drugs in female students of pharmacy," after applying the selection criteria I'll take a sample of 176 students, the instrument was a questionnaire adapted from the Global Youth Tobacco Survey (GYTS), prepared by CEDAR WHO-CDC-2000, version 42 reagents were used. The variables were treated in terms of frequency and percentage, the association between the variables family and friendly relations with the consumption of snuff by Chi-square test was evaluated. Spearman coefficient was used to calculate the significance of correlation between the scores assigned to family and friendly relations with the variable consumption of snuff. Results: there is no significant correlation between the type of family relationships and consumption of snuff in pharmacy students. The students whose friends do not smoke, only $48 \%$ smoked while students with some friends who smoked and those that most of their friends smoke,

Facultad de Enfermería, Universidad Peruana Cayetano Heredia. Lima, Perú.

Instituto Nacional de Salud del Niño. Lima, Perú.

Docente ; ${ }^{\mathrm{b}}$ Lic. Esp. Enf. Madre y Recién nacido ; ${ }^{\mathrm{c}}$ Lic. Esp. Enf Pediátrica ; ${ }^{\mathrm{d}}$ supervisora ; ${ }^{\mathrm{e}}$ Doctora en Salud Pública 
this consumption figure amounted to $70.4 \%$ and $85.7 \%$ respectively. Conclusions: there is no significant correlation between the type of family relationships and consumption of snuff in pharmacy students. If there is a significant relationship between consumption of snuff in pharmacy students and that some or most of their friends smoke.

KEYWORDS: Associated consumption, snuff, students, women, pharmacy.

\section{INTRODUCCIÓN}

El consumo de tabaco es la principal causa prevenible de muerte prematura que tienen actualmente los países desarrollados, y representa una seria amenaza para la salud pública de los países en vías de desarrollo (1).

El consumo de tabaco provoca a nivel mundial más de 4 millones de muertes evitables al año y es el principal factor causante del $30 \%$ de los cánceres, por ello, se ha convertido en un auténtico reto para la gestión en salud y una de las prioridades en las políticas sanitarias internacionales; se estima que hay cerca de 1300 millones de fumadores; el $43 \%$ de los hombres y el $12 \%$ de las mujeres del mundo fuman, aunque hasta la fecha se asociaba en exclusividad a países desarrollados, en la actualidad se calcula que el $84 \%$ de las personas que fuman viven en países en vías de desarrollo (2).

El consumo de cigarrillo representa para algunas mujeres una herramienta de afrontamiento del estrés y la ansiedad, de control del apetito y el peso corporal y en la interacción social como facilitador de las relaciones sociales y en la interacción sentimental-sexual como herramienta de acercamiento y contacto con el sexo opuesto. Además, el consumo de cigarrillos opera como un símbolo de poder y resistencia frente a situaciones asimétricas entre los sexos y de dominación masculina. Un estudio realizado por Jiménez, M. en la Universidad de Granada -España ha revelado que el consumo de cigarrillos ha adquirido para las mujeres fumadoras significados nuevos y específicos en el contexto de sus vidas cotidianas. Así, para las mujeres el fumar afecta su entorno en cuatro dimensiones: emocional, corporal, social y simbólica y como expresión de poder y resistencia (3).

Entre los posibles "factores de riesgo" o de "protección" que determinan el fenómeno del abuso de drogas, la familia juega un importante papel, ya que es un pilar fundamental en los adolescentes, no sólo porque conforma el espacio de socialización por excelencia, sino porque es aquí donde los adolescentes buscan las pautas fundamentales para orientarse en la vida. Desde el nacimiento hasta la adolescencia la familia es un ámbito privilegiado de formación de actitudes, habilidades y valores que permitirán que posteriormente el sujeto afronte una etapa vital decisiva, como es la adolescencia (4).

La relación con los padres es muy importante para adolescentes y jóvenes, estas pueden constituirse en factores protectores frente al consumo de tabaco, estudios revelan que el nivel de comunicación con el padre y con la madre muestra una relación inversa con el consumo diario de tabaco (5).

En relación a los factores de riesgo y protectores relacionados con la familia y los amigos en un estudio realizado en Valparaíso-Chile, los factores de riesgo más importantes fueron: baja autoestima, falta de actividad deportiva, alta concurrencia a fiestas, tener amigos que fumen y en especial su mejor amigo, como también padres y/o familiares fumadores que viven permanentemente con ellos. Entre los factores protectores se destacan: la participación en deportes, buena apreciación de sí mismos, compartir con amigos que no fumen, en especial su mejor amigo, tener padres que no consuman cigarrillos y poseer un buen conocimiento del daño que provoca el tabaquismo para la salud (6).

Un estudio realizado en Chile, arroja datos reveladores respecto a la relación existente entre los altos índices de tabaquismo en los adolescentes y la permisividad de los padres, señalando que el $100 \%$ de los adultos fumadores autorizan a sus hijos para que fumen cuando éstos tienen entre 15 y 18 años $(7,8)$.

El estudio tuvo como objetivo determinar la relación existente entre el consumo de tabaco y otras drogas en mujeres jóvenes universitarias y las características de sus relaciones familiares y de amigos.

\section{MATERIAL Y MÉTODOS}

El estudio fue de tipo, cuantitativo, correlacional, de corte transversal, se realizó en la Facultad de Farmacia de una Universidad Privada de Lima-Perú. Los datos fueron extraídos de una base de datos trabajada por la investigadora para el estudio: "Conocimientos y prácticas de consumo de tabaco y otras drogas en estudiantes de pre grado de una facultad de farmacia-Lima 2008" (9). Los criterios de selección fueron: ser estudiantes de farmacia, de sexo 
femenino, haber firmado el consentimiento informado.

La población estuvo conformada por 314 estudiantes de ambos sexos, 176 estudiantes cumplieron los criterios de inclusión.

En dicho estudio la técnica utilizada fue la encuesta auto-aplicada o por entrevistador y como instrumento una versión adaptada del cuestionario del Global Youth Tobacco Survey (GYTS), elaborado por OMS-CDC-CEDRO en el año 2000 (10), que consta de 92 reactivos , de los cuales para la presente investigación solo se tomaron 42 reactivos, estos reactivos fueron cerrados de opción múltiple y selección única, organizados en las siguientes áreas: datos generales, consumo, relaciones familiares y relaciones amicales. Asimismo se consideraron datos de prevalencia del consumo de alcohol y drogas ilegales. Para la respuesta a relaciones familiares se consideró:1 mala, 2 regular y 3 buena. Tanto para relaciones con el padre, la madre y los hermanos, el puntaje mínimo fue $3(1,1,1)$ y el máximo $9(3,3,3)$, la clasificación fue la siguiente:

$<=4 \quad$ mala (hasta 2 malas y 1 regular)

5 a 7 regular (hasta 2 regulares y 1 buena)

$>7$ buena (desde 2 buenas y 1 regular).

De acuerdo a los datos obtenidos, estos fueron ingresados al programa SPSS versión 19 para realizar el análisis estadístico, obteniéndose los resultados de la investigación de acuerdo a los promedios establecidos en la clasificación de las variables.

Las variables consumo, relaciones familiares y relaciones amicales, fueron tratadas como: dicotómicas y categóricas en términos de frecuencia y porcentaje, para medir la asociación entre las variables relaciones familiares y amicales con el consumo de tabaco, se utilizó la estadística inferencial a través de la Prueba de Chi-cuadrado. Para la correlación entre las puntuaciones asignadas a las relaciones familiares y amicales con la variable consumo de tabaco y otras drogas (indicadores ordinales) se calcularon el coeficiente de correlación de Spearman. Se utilizó un nivel de significancia estadística con valores de $p$ inferior a 0,05 .

La variable consumo de tabaco, se trabajó con frecuencias y porcentajes relacionados a si consume y no consume. Para la variable relaciones familiares y amicales, la relación fue clasificada en buena, regular y mala.

El proyecto de investigación fue aprobado por el Comité Institucional de ética de la Universidad Peruana Cayetano Heredia. En el estudio se consideró el respeto de los principios éticos para la investigación en seres humanos, se dio un trato justo y equitativo, a todos los participantes, se les brindó información irrestricta, su participación fue voluntaria y los beneficios de educación y capacitación por CEDRO, revirtieron en los estudiantes sujetos de investigación.

Tabla 1. Estudiantes de farmacia según edad y ciclo academico de estudios- LIMA- 2007.

\begin{tabular}{lcc}
\hline Edad & Frecuencia & Porcentaje \\
\hline 16 a 18 años & 30 & 17,0 \\
19 a 22 años & 80 & 45,5 \\
23 a 26 años & 36 & 20,6 \\
27 a 30 años & 18 & 10,5 \\
31 a más años & 4 & 2,5 \\
No responde & 7 & 4,0 \\
Ciclo que cursa & & \\
1er y 2do ciclo o 1er año & 35 & 19,9 \\
3er y 4to ciclo o 2do año & 52 & 29,6 \\
5to y 6to ciclo o 3er año & 41 & 23,5 \\
7mo y 8vo ciclo o 4to año & 31 & 17,7 \\
9no o 10mo ciclo o 5to año & 15 & 8,7 \\
No responde & 1 & 0,7 \\
Total & $\mathbf{1 7 5}$ & $\mathbf{1 0 0 , 0}$ \\
\hline
\end{tabular}




\section{RESULTADOS}

El 45,5\% de las estudiantes, pertenecían al grupo etario de 19 a 22 años y al tercer y cuarto ciclo académico de estudios $(29,6 \%)$ (Tabla 1$)$.

La tabla 2, muestra que el mayor porcentaje, corresponde al $66,3 \%$ de estudiantes de farmacia que han consumido tabaco alguna vez en la vida.

En la tabla 3 observamos que un 59,4\% de estudiantes mujeres de farmacia manifiestan tener buenas relaciones familiares.

Observamos en la tabla 4, que un 50\% de las alumnas califican la relación entre sus padres como mala, han fumado, durante los últimos 30 días, entre 10 y 29 días, este porcentaje en los grupos correspondiente a relaciones entre los padres regular y buena fueron de 7,5\% y $4,1 \%$ respectivamente. Las diferencias resultaron estadísticamente significativas $(\mathrm{p}=0,088)$.

Además se ha observado que existe correlación entre

Tabla 2. Prevalencia de vida de consumo de tabaco en estudiantes de farmacia. Lima, Perú 2007.

\begin{tabular}{ccc}
\hline $\begin{array}{c}\text { Consumo de tabaco alguna vez } \\
\text { en la vida }\end{array}$ & Frecuencia & Porcentaje \\
\hline Si consume & 116 & 66,3 \\
No consume & 50 & 28,6 \\
No responde & 9 & 5,1 \\
Total & 175 & 100,0 \\
\hline
\end{tabular}

Tabla 3. Relaciones familiares en estudiantes mujeres de farmacia. Lima, Perú 2007.

\begin{tabular}{lcc}
\hline & Frecuencia & Porcentaje \\
\hline MALA & 11 & 6,3 \\
REGULAR & 56 & 32,0 \\
BUENA & 104 & 59,4 \\
Total & 171 & 97,7 \\
No responde & 4 & 2,3 \\
Total & 175 & 100,0 \\
\hline
\end{tabular}

Tabla 4. Relacion entre padres y consumo de tabaco actual en estudiantes mujeres de farmacia. Lima, Perú 2007.

\begin{tabular}{|c|c|c|c|c|c|c|c|c|}
\hline \multirow{2}{*}{$\begin{array}{l}\text { Relación } \\
\text { entre padres }\end{array}$} & \multicolumn{7}{|c|}{ Consumo de tabaco actual -último mes } & \multirow[t]{2}{*}{ Total } \\
\hline & $\begin{array}{l}\text { Ningún } \\
\text { día }\end{array}$ & $\begin{array}{l}1 \text { a } 2 \\
\text { días }\end{array}$ & $\begin{array}{l}3 \text { a } 5 \\
\text { días }\end{array}$ & $\begin{array}{l}6 \text { a } 9 \\
\text { días }\end{array}$ & $\begin{array}{c}10 \text { a } 19 \\
\text { días }\end{array}$ & $\begin{array}{c}20 \text { a } 29 \\
\text { días }\end{array}$ & $\begin{array}{l}\text { Todos los } \\
\text { días }\end{array}$ & \\
\hline \multirow[t]{2}{*}{ Mala } & 2 & 0 & 0 & 0 & 1 & 1 & 0 & 4 \\
\hline & $50,0 \%$ & $0,0 \%$ & $0,0 \%$ & $0,0 \%$ & $25,0 \%$ & $25,0 \%$ & $0,0 \%$ & $100,0 \%$ \\
\hline \multirow[t]{2}{*}{ Regular } & 30 & 4 & 1 & 2 & 2 & 1 & 0 & 40 \\
\hline & $75,0 \%$ & $10,0 \%$ & $2,5 \%$ & $5,0 \%$ & $5,0 \%$ & $2,5 \%$ & $0,0 \%$ & $100,0 \%$ \\
\hline \multirow[t]{2}{*}{ Buena } & 67 & 15 & 7 & 2 & 3 & 1 & 1 & 96 \\
\hline & $69,8 \%$ & $15,6 \%$ & $7,3 \%$ & $2,1 \%$ & $3,1 \%$ & $1,0 \%$ & $1,0 \%$ & $100,0 \%$ \\
\hline \multirow[t]{2}{*}{ Total } & 99 & 19 & 8 & 4 & 6 & 3 & 1 & 140 \\
\hline & $70,7 \%$ & $13,6 \%$ & $5,7 \%$ & $2,9 \%$ & $4,3 \%$ & $2,1 \%$ & $0,7 \%$ & $100,0 \%$ \\
\hline
\end{tabular}


Tabla 5. Relación entre consumo de tabaco de amigos y consumo de tabaco de las estudiantes mujeres de farmacia. Lima, Perú 2007.

\begin{tabular}{cccc}
\hline $\begin{array}{c}\text { CONSUMO DE TABACO } \\
\text { EN AMIGOS }\end{array}$ & \multicolumn{2}{c}{$\begin{array}{c}\text { CONSUMO DE TABACO EN } \\
\text { ESTUDIANTES }\end{array}$} & Total \\
\hline \multirow{2}{*}{ Ninguno fuma } & $\mathrm{Si}$ & No & $\mathrm{Si}$ \\
Algunos fuman & & & \\
& 12 & 13 & 25 \\
La mayoría de ellos fuma & $48,0 \%$ & $52,0 \%$ & $100,0 \%$ \\
& 69 & 29 & 98 \\
Todos fuman & $70,4 \%$ & $29,6 \%$ & $100,0 \%$ \\
& 30 & 5 & 35 \\
Total & $85,7 \%$ & $14,3 \%$ & $100,0 \%$ \\
& 4 & 2 & 6 \\
& $66,7 \%$ & $33,3 \%$ & $100,0 \%$ \\
& 115 & 49 & 164 \\
& $70,1 \%$ & $29,9 \%$ & $100,0 \%$ \\
\hline
\end{tabular}

relaciones familiares y la intención de fumar durante los próximos meses. El valor negativo del coeficiente $(\mathrm{R}=-$ $0,155)$ indica que la correlación es inversa, es decir, en la medida que mejoran la relaciones familiares disminuye la intención de fumar en los próximos meses, siendo la correlación estadísticamente significativa $(\mathrm{p}=0,043)$.

Existe correlación entre relaciones familiares y el número de cigarrillos fumados por día en el último mes. El valor negativo del coeficiente $(\mathrm{R}=-0,215)$ indica que la correlación es inversa, es decir, en la medida que mejoran la relaciones familiares disminuye el número de cigarrillos fumados en el último mes, siendo la correlación estadísticamente significativa $(p=0,005)$.

En relación a las amistades, en la tabla 5, se puede observar que en aquellas estudiantes cuyas amistades no fuman; solo un $48 \%$ alguna vez ha fumado aunque sea una o dos pitaditas, mientras que en quienes algunas de sus amistades fuman este porcentaje es de $70,4 \%$ y en aquellos que la mayoría de sus amistades fuman un $85,7 \%$ ha fumado. Este porcentaje en quienes todas sus amistades fuman es también alto $(66,7 \%)$. Las diferencias del grupo con amistades que no fuman respecto al resto, en lo relacionado al consumo de tabaco, es estadísticamente significativa $(\mathrm{p}=0,019)$.

\section{DISCUSION}

El incremento del consumo de tabaco en la población juvenil constituye un problema necesario de ser abordado por las autoridades a nivel nacional, pues sus indicadores revelan cifras alarmantes en el consumo actual (10). Pero también constituyen una preocupación prioritaria para la Universidad, debido a que estudios evidencian que estudiantes universitarios tienen elevados índices de consumo de tabaco $(5,11)$, muchos de ellos iniciados en el colegio y fortalecido en los grupos universitarios. Zárate $\mathrm{M}$, en su estudio refiere que los hábitos de consumo en estudiantes universitarios peruanos del área de salud son poco conocidos pero importantes por su impacto en la formación de recursos que prestarán servicios a la población expuesta a este grave problema de salud pública (11).

La importancia del presente trabajo en el abordaje a un grupo de jóvenes: mujeres estudiantes de farmacia, porque estudios demuestran que actualmente el consumo de tabaco en mujeres se está incrementando y la diferencia de género es mínima (12), un estudio de OMS/OPS revela que a pesar de lo que conocemos hoy acerca del tabaco, el consumo sigue aumentando en todo el mundo (13). La epidemia sigue en expansión, especialmente en los países menos desarrollados.

En la actualidad en el Perú, según reportaje de DEVIDA en 2005, aproximadamente 2 millones de peruanos mayores de 15 años fuman, con una prevalencia de consumo de vida (alguna vez en su vida) del 60,5\%; en el año, la prevalencia de consumo para los varones alcanza el 75,9\% y para las mujeres un $60,3 \%$. Si vemos la prevalencia por mes se reporta un $39,3 \%$ en los hombres y un $16,7 \%$ para las mujeres (14). 
El último estudio epidemiológico a nivel nacional realizado por CEDRO en el año 2005, demostró que el consumo de tabaco sigue ocupando el segundo lugar en el consumo de drogas en el Perú, además puntualiza que el $75 \%$ de la población está expuesta a ser envuelta en el consumo de tabaco y el $60,5 \%$ ha consumido esta sustancia alguna vez en su vida (15).

En este contexto se desarrolló el presente estudio en una población de estudiantes mujeres de farmacia, donde la mayoría de ellas se encontraban en el grupo etario de 19 a 22 años y cerca de la tercera parte en el segundo año académico de estudios, según se muestra en la tabla 1.

$\mathrm{Al}$ indagar sobre el consumo de tabaco de las estudiantes de farmacia encontramos que en lo referente a prevalencia de vida de consumo de tabaco, que un $66,3 \%(116 / 175)$ han consumido tabaco alguna vez en su vida, mientras $28,6 \%$ afirma no haber consumido nunca (Tabla 2).

Resultados similares encontramos en el estudio realizado por CEDRO, sobre el consumo de drogas legales e ilegales en estudiantes universitarios, provenientes de universidades públicas y privadas en Lima, donde encontró que la prevalencia de vida, alcanza el 77,5\% (16), que es mucho mayor al encontrado en el anterior estudio de consumo de drogas también realizado por esta institución con jóvenes entre los 16 y 25 años, donde se encontró que esta prevalencia era de $60,4 \%$ (17).

Esto nos evidencia una tendencia al incremento de consumo de tabaco en la población juvenil. Al respecto CEDRO en su estudio, comenta que el consumo de tabaco podría bien ser resultado del efecto de modelos grupales que fuman en un ambiente donde el joven universitario busca aproximarse al grupo, sin dejar de lado el efecto de las características adictivas de la propia sustancia (16). En el caso del alcohol y del tabaco, se hace presente también la enorme aceptación social que tienen ambas sustancias y el refuerzo que este recibe por parte de importantes campañas publicitarias que promueven su consumo.

Estudios anteriores realizados a nivel de la población en general encuentran una prevalencia para el año 2000 de $61 \%$ en los varones y $46 \%$ en las mujeres (17); sin embargo estudios como el realizado en escolares en España encuentran un mayor consumo de tabaco en mujeres con una diferencia del 7\% (7).

A diferencia otro estudio encuentra un consumo en similares proporciones para ambos sexos, como el realizado por
Valdés, en México (2002), que nos revela un consumo en escolares donde no existen diferencias estadísticamente significativas entre el consumo de cigarros por los hombres $(19,6 \%)$ y por las mujeres $(19,1 \%)(8)$.

Con respecto al consumo actual, en el presente estudio se ha encontrado en las estudiantes de farmacia, un consumo de $34,3 \%$.

El consumo actual de tabaco encontrado en el estudio, es menor a las cifras de un estudio realizado en Chile, en la ciudad de Valparaíso en adolescentes, donde un porcentaje significativo de jóvenes correspondiente al 43,3\% consumía tabaco actualmente (18) y mayor al de consumo encontrado en un estudio realizado en Asunción en el 2007 a un grupo de estudiantes de enfermería y obstetricia, donde del total de estudiadas $16,8 \%$ respondieron que fuman actualmente, de estos el $27 \%$ lo hace diariamente y $73 \%$ ocasionalmente (19).

Un estudio muestra que en otros países, el consumo de tabaco es la sustancia más prevalente entre las chicas que entre los chicos y además fuman con mayor intensidad. Desde hace varios años las investigaciones confirman que las mujeres se han incorporado con fuerza al hábito de fumar, llegando a superar a los hombres (20).

Las estudiantes de farmacia al igual que muchas jóvenes del país y de otros países tienen múltiples razones para el consumo de tabaco, estas razones pueden estas asociadas como dice Jiménez en su estudio (3): fumar para controlar el peso, la utilización del cigarrillo como instrumento regulador del apetito está ligada al modelo corporal femenino imperante en Occidente, asentado en la delgadez, Otro sentido relevante está relacionado con la atracción sexual y la seducción, si bien éste es más importante durante la adolescencia y la juventud.

Si analizamos el ambiente alrededor de la universidad, podemos comprobar que existen numerosos lugares de expendio de comidas, alcohol y tabaco; donde los estudiantes en sus momentos "libres" acuden en grupo y pueden consumir estas sustancias sin mayor control de autoridades y sin ningún respeto de las normas establecidas a nivel de gobiernos locales.

En relación al consumo de tabaco en familiares encontramos que es el padre el que presenta el mayor consumo $16,3 \%$ y el $5,9 \%$ de los hermanos. Sin embargo al indagar sobre el consumo de sus amigos encontramos que el $59,5 \%$ responde que algunos fuman y el $21,6 \%$ que la 
mayoría de ellos fuman, es decir que las jóvenes en un $81,1 \%$, están rodeadas de consumidores ocasionales y/o frecuentes de tabaco, con los que pasa la mayor parte de su tiempo en la universidad.

Se ha asociado la edad de inicio del consumo de tabaco en las estudiantes y la relación entre sus padres y se encontró que del $100 \%$ de las estudiantes que calificaron como malas las relaciones entre sus padres, probaron su primer cigarrillo entre los 14 y 15 años, mientras que aquellas que calificaban las relaciones de sus padres como regulares o buenas lo hicieron cuando tenían 16 años o más. Las diferencias resultaron significativas.

El hecho de encontrar un bajo consumo por parte de los padres y hermanos, o sea a nivel del núcleo familiar y un elevado consumo según refieren las estudiantes en su grupo de amigos más cercanos, nos debe llevar a pensar en planificar estrategias de prevención dirigidas más al grupo de pares. Al respecto un estudio realizado en el país hace énfasis sobre la importancia de la percepción de tabaquismo en el mejor amigo, hermano o compañero son de manera independiente los factores asociados con el incremento del riesgo de consumo de tabaco (21), de igual manera un estudio realizado en Argentina reporta que la mayoría de jóvenes fumadores señaló a la imitación de esa conducta en los amigos como el motivo predominante para iniciarse en el hábito de consumo de tabaco (22).

En la tabla 3, se abordan las relaciones familiares entre las estudiantes, padres y hermanos y un 59,4\% refieren tener buenas relaciones familiares.

Estudios similares refieren que, en cuanto a la relación y comunicación con los padres, la mayoría de los entrevistados mencionó tener mejor relación con la madre que con el padre, posiblemente porque en las familias en cuestión aún se ejercen los roles tradicionales de género, lo que implica que la madre sea afectivamente más cercana que el padre. Los entrevistados de mayor edad, que dijeron tener más dificultad para relacionarse y comunicarse con sus padres, son los que presentan complicaciones académicas y sociales más significativas, además de mayor consumo de tabaco y alcohol (23).

La tabla 4, muestra que el 74,5\% de estudiantes que refieren tener buenas relaciones familiares han consumido tabaco alguna vez en la vida.

Aquí es importante reflexionar que cuando la comunicación del adolescente o joven con sus padres falla, estos buscan otros referentes para la comunicación en el mejor de los casos pueden ser los hermanos, pero si estos tampoco asumen ese rol, los adolescente y jóvenes terminan buscando apoyo en los amigos. Gómez (2008), nos dice en su estudio: Dicha dificultad para relacionarse con sus padres radica básicamente en una escasa o nula comunicación con el padre (éste no era mencionado al preguntarles por su familia, no lo nombraban hasta que se les preguntaba específicamente por él) y una comunicación insuficiente con la madre (4).

En estos casos los adolescentes manifiestan conductas oposicionistas a las instrucciones de los padres y prefieren autoafirmarse con otros grupos sociales - como los amigos - o experimentando conductas que pueden ser riesgosas.

$\mathrm{Al}$ analizar las relaciones de la estudiante con el padre y la madre, con el consumo de tabaco, no se encontró asociación significativa, situación diferente fue la encontrada en otro estudio en donde el $80 \%$ dijo tener una muy buena/ relación con el padre y ésta se asoció de manera inversa con el riesgo de haber consumido tabaco durante el último año de vida (a mejor relación menor consumo. El 93\% declaró tener una muy buena/relación con su madre, pero ésta no se asoció en forma significativa con el consumo de tabaco durante el último año de vida $(p>0,05)(25)$.

Se encontró también, que el $50 \%$ de las estudiantes que califican la relación entre sus padres como mala han fumado durante el mes entre 10 a 29 días, mientras que las estudiantes que manifestaron relaciones entre sus padres regulares y buenas lo hicieron en un 7,5\% y 4,1\% respectivamente.

Un estudio realizado por Sanz, concluye en sus resultados, que la presencia de conflicto entre los padres se asociaba a una mayor presencia de consumo de sustancias entre los/as hijos/as. En todos los indicadores de consumo, las variables asociadas a la valoración del conflicto marital, resultaron en mayor o menor medida asociadas a la mayor frecuencia de consumo en los hijos (24).

Andrade, en su estudio indica, que los conflictos en la familia, el hecho de permanecer más tiempo con los amigos que con la familia, el vivir con un solo padre o en hogares reconstituidos son indicadores significativos de uso de drogas, consumo de alcohol y tabaco en los adolescentes (25).

Sobre las relaciones amicales y consumo de tabaco se encontró que las estudiantes cuyas amistades no fuman, 
solo un $48 \%$ fumaba, mientras que las estudiantes con algunas de sus amistades que fumaban y aquellas que la mayoría de sus amistades fumaba, esta cifra de consumo ascendió a 70,4\% y 85,7\% respectivamente (Tabla 5).

En un estudio realizado por CEDRO, se encontró que el ofrecimiento de cigarrillos de tabaco es frecuente en las ciudades del país, y es realizado principalmente por amigos (35 a 65\%) o familiares cercanos (3,1 a 7\%) (10).

\section{REFERENCIAS BIBLIOGRÁFICAS}

1. Prat-Marín A, Fuentes A, Sanz P, et al. Epidemiología del tabaquismo en los estudiantes de ciencias de la salud. Rev Saúde Pública. 1994; 28 (2):100-6.

2. Banco Mundial; OPS/OMS. La epidemia de tabaquismo: Los gobiernos y los aspectos económicos del control del tabaco. Publicación Científica N ${ }^{\circ} 577$. Washington DC: Banco Mundial; OPS/OMS; 2000.

3. Jiménez ML. Mujeres y tabaco: La feminización del consumo de cigarrillos en España Tesis Doctoral. Granada, España: Departamento de Antropología Social, Universidad de Granada; 2007.

4. Sanz M, Martínez A, Iraugi I, Galìndez E, Muñoz A, Cosgaya L. Influencia de la familia sobre el consumo de drogas en jóvenes. Psicoteca. 2005. (Citado en noviembre del 2015) Disponible en: http://paginaspersonales.deusto. es/matute/psicoteca/articulos/Sanzetal05.htm

5. Martínez-Hernáez A, Marí-Klose M, Julià A, Escapa $\mathrm{S}$, Marí-Klose P, DiGiacomo S. Consumo diario de tabaco en la adolescencia, estados de ánimo negativos y rol de la comunicación familiar. GacSanit. 2012; 26(5): 421-428. http://dx.doi.org/10.1016/j.gaceta.2011.09.030

6. González L, Berger k. Consumo de tabaco en adolescentes: factores 0 de riesgo y factores protectores. Cienc enferm. 2002 (citado el 10 de agosto del 2014) ; 8( 2 ): 27-35. Disponible en: http://www.scielo.cl/scielo.php?script=sci arttext\&pid=S0717-95532002000200004\&lng=es. http:// dx.doi.org/10.4067/S0717-95532002000200004

7. Sociedad Española de especialistas en tabaco. Recursos para los padres para evitar que los jóvenes fumen. Resumen de las XI Jornadas de Tabaquismo en España. Madrid: Sociedad Española de especialistas en tabaco; Mayo 2012. (citado el 10 de agosto del 2014) Disponible en: http://www. sedet.es/webcms/index.php?menu=noticias\&submenu= ver_noticia\&id_noticia $=11542$

8. Valdés R, Micher J. Tendencias del consumo de tabaco entre alumnos de nuevo ingreso a la Universidad Nacional Autónoma de México, 1989 a 1998. Salud pública Méx. 2002; 44(1):S44-S53.

9. Danjoy D, Ferreira PS, Pillon SC. Conocimientos y prácticas sobre el consumo de tabaco en estudiantes de pregrado de farmacia, Lima, Perú. Rev Latino-Am Enfermagem. 2010 (citado el 10 de agosto del 2014); 18: 582-588. Disponible en:http://www.scielo.br/scielo. p h p ? s c ript $=$ s c i a r t text \& pid=S 0104 $11692010000700014 \& \operatorname{lng}=$ en\&nrm=iso

10. CEDRO. El problema de las drogas en el Perú. Lima: CEDRO; Junio del 2005.

11. Zárate M, Zavaleta A, Danjoy D, et al. Prácticas de consumo de tabaco y otras drogas en estudiantes de ciencias de la salud de una Universidad Privada de Lima Perú. Invest educ enferm. 2006; (24) 2: 72-81.

12. Dejar de fumar: El tabaquismo es una enfermedad que podemos combatir. Las jovenes y el consumo del tabaco. Disponible en: http://dejar-de-fumar-tabaquismo.blogspot. pe/2006/05/las-jovenes-y-el-consumo-del-tabaco.html

13. OMS/OPS. Encuesta mundial sobre tabaquismo en la juventud: Resultados en las Américas. Boletín Epidemiológico. 2002; 23(2).

14. Centro para el Control y la Prevención de Enfermedades. Las mujeres y el tabaquismo. Tabaquismo. Informe de la Dirección General de Salud Pública. Atlanta: Centro para el Control y la Prevención de Enfermedades.

15. Castro de la Mata R, Zavaleta A. Consumo de tabaco y la influencia de los amigos. Monografía de Investigación N²4. Lima, Perú: CEDRO; 2006.

16. Centro de Información y Educación para la Prevención del Abuso de Drogas El problema de las drogas en el Perú. Lima, Perú: CEDRO; 2003.

17. Poletto L, Pezzoto SM, Morini J,y Andrade J. Prevalencia del hábito de fumar en jóvenes y sus padres: asociaciones relevantes con educación y ocupación. Rev Saúde Pública. 1991;25 (5): 388-393.

18. González L, Berger K. Consumo de tabaco en adolescentes: Factores de riesgo y protectores. Cienc Enferm. 2002;8(2): 27-35.

19. Morel J. Conocimientos, actitudes y prácticas sobre tabaquismo en estudiantes de Enfermería y Obstetricia del Instituto Dr. Andrés Barbero- Año2007. Mem Inst Investig Cienc Salud. 2008;4(2):38-47.

20. Gómez E. Adolescencia y familia: revisión de la relación y la comunicación como factores de riesgo o protección. Revista Intercontinental de Psicología y Educación. 2008;10(2):105-122.

21. Colegio Médico de Perú. Guía Nacional de abordaje Técnico al Tabaquismo.1era ed. Lima., Perú: Colegio Médico del Perú; 2010. (citado el 10 de agosto del 2014) Disponible en: http://bvs.minsa.gob.pe/local/MINSA/1122 GRAL1364.pdf

22. Bolzan A, Peleteiro R. Tabaquismo durante la adolescencia temprana: estudio en escolares argentinos. J Pediatr. (Rio J.). $2003 ; 79(5)$ : 461-466.

23. Gómez E. Adolescencia y familia: revisión de la relación y la comunicación como factores de riesgo o protección. Revista Intercontinental de Psicología y Educación. 2008; 10(2):105-122. (citado el 10 de agosto del 2014) Disponible en:http://redalyc.uaemex.mx/ pdf/802/80212387006.pdf

24. Andrade P. El ambiente familiar del adolescente. Tesis de Doctorado. Ciudada de Mexico, Mexico: Facultad de Psicología, Universidad Nacional Autónoma de México; 
1998.

25. Glavic M. Protectores del consumo de drogas en población adolescente. Tesis para optar el grado de magister en Sociología. Santiago, Chile: Pontificia Universidad Católica de Chile; 2008.

\section{Correspondencia:}

Delia Danjoy León

Correo electrónico: delia.danjoy@upch.pe

Fecha de Recepción: 03 de setiembre del 2015.

Fecha de aceptación: 12 de octubre del 2015. 